\begin{tabular}{c|c|c|} 
SELECCIONES MATEMÁTICAS \\
Universidad Nacional de Trujillo \\
ISSN: $2411-1783$ (Online) \\
2019; Vol.06(2): $311-319$.
\end{tabular}

COMMUNICATION

\title{
Sobre un álgebra de matrices sin matrices.
}

\section{On a matrix álgebra without matrices.}

\author{
Edgar Vera Saravia*(iD
}

Received, Set. 11, 2019

Accepted, Nov. 20, 2019

How to cite this article:

Vera, E. Sobre un álgebra de matrices sin matrices. Selecciones Matemáticas. 2019; 6(2):311-319. http: //dx. doi .org/ 10.17268 /sel.mat.2019.02.17

\section{Resumen}

Ampliamos la introducción al álgebra geométrica bidimensional AG(2) presentada en el Volumen 05(02) de SELECCIONES MATEMÁTICAS. Se trata de una introducción amigable a un tema de interés común para docentes y estudiantes universitarios de Física y Matemática.

Palabras clave: Álgebra geométrica, encaje de espacios vectoriales, encaje de álgebras reales.

\begin{abstract}
We enlarge a previus introduction to the two-dimensional geometric algebra published in Volume 05(02) of SELECCIONES MATEMÁTICAS. It is a friendly introduction of a theme of common interest for university proffessors and students of physics and mathematics.
\end{abstract}

Keywords: Geometric algebra, nested of real vector spaces, nested of algebras.

1. Introducción. Recordemos que el proceso de algebrizar el análisis y la geometría lo inició Descartes en 1637, con la geometría analítica presentada en un apéndice de su libro El discurso del método. Al enterarse de esto Leibniz le manifiesta a Huygens, en 1679:
la Física no podrá avanzar más a no ser que se encuentre un nuevo método de análisis más geométrico, que permita expresar y operar con direcciones tan directamente como el álgebra (de los números reales) representa y opera con las magnitudes (el concepto de longitud en la geometría analítica) ${ }^{1}$

La intuición de Leibniz, sobre un modelo matemático apropiado para el desarrollo de la Física, encontró una respuesta en el Álgebra Geométrica creada por Clifford entre los años 1873 y 1879, conjugando el álgebra de los cuaterniones de Hamilton (1843) y el álgebra exterior de Grassmann (1844).

Debemos recordar que Hamilton, al descubrir en 1835 que los complejos le permitían algebrizar el proceso geométrico de rotación en $\mathbb{R}^{2}$, buscó denodadamente por más de siete años los cuaterniones que permiten algebrizar, del mejor modo, las rotaciones en $\mathbb{R}^{3}$.

En estas notas tratamos el caso bidimensional. Posteriormente incluiremos los demás espacios del siguiente encaje de $\mathbb{R}$-espacios vectoriales:

$$
\mathbb{R}^{2}<\mathbb{R}^{3}<\mathbb{R}^{4}
$$

\footnotetext{
${ }^{*}$ Facultad de Ciencias Matemáticas - UNMSM, Av. Venezuela s.n., Lima-Perú. (everas@unmsm.edu • pe)

${ }^{1}$ traducción del autor
} 
El objetivo final es construir estructuras de álgebra geométrica asociadas a estos tres espacios y los dos siguientes encajes de álgebras geométricas:

$$
A G(2)<A G(3)<A G(3,1)>A G(1,1)
$$

de tal modo que estas estructuras de álgebra geométrica expliciten respectivamente: De un lado, que los dos primeros $\mathbb{R}$-espacios vectoriales del encaje (1.1) adquieren la métrica Euclideana y, de otro, el primero y último $\mathbb{R}$-espacios vectoriales del encaje (1.1) adquieren la métrica de Minkowski.

2. Un álgebra real de matrices reales. Repensamos el álgebra no conmutativa de matrices $2 \times 2$ con entradas reales, $\mathbb{R}^{2 \times 2}$, adaptando convenientemente el contexto que Wolfgang Pauli propuso para $\mathbb{C}^{2 \times 2}$ en la década de los años 20 del siglo pasado.

El aporte de Pauli sugiere considerar, para el caso que nos interesa, las matrices

$$
\sigma_{1}=\left[\begin{array}{ll}
0 & 1 \\
1 & 0
\end{array}\right] \quad \text { y } \quad \sigma_{2}=\left[\begin{array}{cc}
1 & 0 \\
0 & -1
\end{array}\right]
$$

que llamaremos $\mathbb{R}$-matrices de Pauli, las mismas que tienen propiedades realmente maravillosas:

Construyamos la tabla multiplicativa de estas matrices

\begin{tabular}{c|cc|cc} 
& $\sigma_{1}$ & & $\sigma_{2}$ & \\
\hline$\sigma_{1}$ & 1 & 0 & 0 & -1 \\
& 0 & 1 & 1 & 0 \\
\hline$\sigma_{2}$ & 0 & 1 & 1 & 0 \\
& -1 & 0 & 0 & 1
\end{tabular}

Denotando con $\sigma_{0}$ la matriz identidad, esta tabla permite verificar las identidades

$$
\sigma_{1} \sigma_{1}=\sigma_{2} \sigma_{2}=\sigma_{0} \quad \text { y } \quad \sigma_{1} \sigma_{2}=-\sigma_{2} \sigma_{1}
$$

conocidas como las condiciones de Grassmann-Clifford matriciales.

La tabla (2.1) también permite ver que toda matriz $A \in \mathbb{R}^{2 \times 2}$ se escribe, de modo único, en la forma

$$
A=\left(\begin{array}{ll}
a & c \\
b & d
\end{array}\right)=\frac{a+d}{2} \sigma_{0}+\frac{b+c}{2} \sigma_{1}+\frac{a-d}{2} \sigma_{2}+\frac{b-c}{2} \sigma_{1} \sigma_{2}
$$

La idea es dejar de usar matrices explítamente y expresar todo en términos de $\sigma_{0}, \quad \sigma_{1}$ y $\quad \sigma_{2}$. Comenzaremos reemplazando la tabla (2.1) por la tabla

\begin{tabular}{c|c|c} 
& $\sigma_{1}$ & $\sigma_{2}$ \\
\hline$\sigma_{1}$ & $\sigma_{0}$ & $\sigma_{1} \sigma_{2}$ \\
\hline$\sigma_{2}$ & $-\sigma_{1} \sigma_{2}$ & $\sigma_{0}$
\end{tabular}

Esta tabla es equivalente a las condiciones de Grassmann-Clifford (2.2).

Conviene extender la tabla (2.4): Sin emplear explícitamente matrices y usando solamente la asociatividad del producto obtenemos la tabla multiplicativa:

\begin{tabular}{c|c|c|c} 
& $\sigma_{1}$ & $\sigma_{2}$ & $\sigma_{1} \sigma_{2}$ \\
\hline$\sigma_{1}$ & $\sigma_{0}$ & $\sigma_{1} \sigma_{2}$ & $\sigma_{2}$ \\
\hline$\sigma_{2}$ & $-\sigma_{1} \sigma_{2}$ & $\sigma_{0}$ & $-\sigma_{1}$ \\
\hline$\sigma_{1} \sigma_{2}$ & $-\sigma_{2}$ & $\sigma_{1}$ & $-\sigma_{0}$
\end{tabular}

Tabla para abstraer el producto de matrices

Todo lo anterior nos permite considerar $\mathbb{R}^{2 \times 2}$ como un $\mathbb{R}$-espacio vectorial de polinomios en las variables $\sigma_{1}$ y $\sigma_{2} ;$ precisamente,

$$
\mathbb{R}^{2 \times 2}=\left\{a_{0} \sigma_{0}+a_{1} \sigma_{1}+a_{2} \sigma_{2}+a_{12} \sigma_{1} \sigma_{2} ; \quad a_{J} \in \mathbb{R}\right\}
$$


provisto de un producto que es una variante del conocido producto de polinomios en el sentido que se procesa utilizando la distributividad y la asociatividad pero no la conmutatividad, en lugar de la conmutatividad se emplea la tabla (2.5).

El contexto matemático usual se dice que el producto considerado es una aplicación $\mathbb{R}$-bilineal asociativa y no conmutativa,

$$
\mathbb{R}^{2 \times 2} \times \mathbb{R}^{2 \times 2} \longrightarrow \mathbb{R}^{2 \times 2}
$$

que se procesa utilizando la tabla (2.5).

El lector interesado puede verificar el proceso del nuevo producto en este momento y luego comparar con lo que presentaremos en la siguiente sección.

Como un primer beneficio de lo tratado anteriormente tenemos que existe un isomorfismo

$\mathbb{R}^{2 \times 2} \equiv \mathbb{C} \oplus \mathbb{R}^{2}$, como $\mathbb{R}$-espacios vectoriales: De modo más preciso, $\mathbb{R}^{2 \times 2}$ contiene copias isomorfas de:

- $\mathbb{R}^{2}$, como $\mathbb{R}$-subespacio vectorial: Asociar su base canónica $\left\{e_{1}, e_{2}\right\}$ con $\left\{\sigma_{1}, \sigma_{2}\right\}$.

- $\mathbb{C}$, como $\mathbb{R}$-subálgebra: Asociar su base canónica $\{1, i\}$ con $\left\{\sigma_{0}, \sigma_{1} \sigma_{2}\right\}$ y verificar que el producto introducido en la versión polinomial de $\mathbb{R}^{2 \times 2}$ extiende el producto de $\mathbb{C}$.

Todo esto mejora grandemente las cosas porque permitirá definir un producto de vectores de $\mathbb{R}^{2}$ y con ello algebrizar resultados geométricos y obtener un tratamiento unificado de álgebra, análisis y geometría bidimensionales.

En resumen, presentaremos amigablemente el álgebra geométrica $\mathrm{AG}(2)$ como una de las "álgebra de matrices sin matrices" creadas por Clifford en 1878 y rescatadas por David Hestenes en los años 60 del siglo pasado (ver [2] y [3]).

\section{El álgebra geométrica bidimensional AG(2).}

Definition 3.1. El álgebra geométrica euclideana bidimensional $A G(2)$ será el siguiente $\mathbb{R}$-espacio vectorial de polinomios reales, en las variables $u_{1}$ y $u_{2}$,

$$
A G(2):=\left\{a_{0}+a_{1} u_{1}+a_{2} u_{2}+a_{3} u_{1} u_{2} ; \quad a_{j} \in \mathbb{R} .\right\}
$$

Sus elementos serán llamados números geométricos.

$A G(2)$ estará provisto de un producto asociativo pero no conmutativo, llamado producto euclideano, determinado por una aplicación $\mathbb{R}$-bilineal asociativa pero no conmutativa

$$
A G(2) \times A G(2) \longrightarrow A G(2)
$$

que se procesa utilizando la distributividad, la asociatividad y la siguiente tabla en lugar de la conmutatividad

\begin{tabular}{c|c|c|c}
$l$ & $u_{1}$ & $u_{2}$ & $u_{1} u_{2}$ \\
\hline$u_{1}$ & $l$ & $u_{1} u_{2}$ & $u_{2}$ \\
\hline$u_{2}$ & $-u_{1} u_{2}$ & $l$ & $-u_{1}$ \\
\hline$u_{1} u_{2}$ & $-u_{2}$ & $u_{1}$ & -1
\end{tabular}

Tabla del producto euclideano de $A G(2)$

La validez de la Tabla (3.1) es equivalente a pedir que $\quad u_{1} \quad$ y $\quad u_{2}$ cumplan las condiciones de GrassmannClifford vectoriales:

$$
u_{1} u_{1}=u_{2} u_{2}=1 \quad \text { y } \quad u_{1} u_{2}+u_{2} u_{1}=0 .
$$

Luego veremos que estas condiciones de Grassmann-Clifford (3.2) corresponden al modo algebraico de expresar que $\left\{u_{1}, u_{2}\right\}$ es una base ortonormal.

Reiteramos sobre las operaciones de $\mathrm{AG}(2)$ : Su estructura vectorial nos permite sumar números geométricos como polinomios pero, para multiplicar números geométricos debemos tener cuidado ya que se trata de un proceso similar al producto de polinomios, en el sentido que sólo podemos utilizar la distributividad y la asociatividad pero no la conmutatividad, en su lugar debemos utilizar la tabla (3.1). Los siguientes cálculos explicitan lo comentado: Dados 


$$
A=a_{0}+a_{1} u_{1}+a_{2} u_{2}+a_{3} u_{1} u_{2}, \quad B=b_{0}+b_{1} u_{1}+b_{2} u_{2}+b_{3} u_{1} u_{2} \in A G(2)
$$

se tiene

$$
A+B=\left(a_{0}+b_{0}\right)+\left(a_{1}+b_{1}\right) u_{1}+\left(a_{2}+b_{2}\right) u_{2}+\left(a_{3}+b_{3}\right) u_{1} u_{2} \in A G(2) \quad \text { y }
$$

donde, utilizando la tabla del producto euclideano (3.1),

$$
\begin{aligned}
& c_{0}=a_{0} b_{0}+a_{1} b_{1}+a_{2} b_{2}-a_{3} b_{3} \\
& c_{1}=a_{0} b_{1}+a_{1} b_{0}+a_{3} b_{2}-a_{2} b_{3} \\
& c_{2}=a_{0} b_{2}+a_{2} b_{0}+a_{1} b_{3}-a_{3} b_{1} \\
& c_{3}=a_{0} b_{3}+a_{3} b_{0}+a_{1} b_{2}-a_{2} b_{1} .
\end{aligned}
$$

De otro lado, definiendo:

$$
A G^{+}(2):=\left\{a_{0}+a_{3} u_{1} u_{2} ; \quad a_{j} \in \mathbb{R}\right\} \quad \text { y } \quad A G_{1}(2):=\left\{a_{1} u_{1}+a_{2} u_{2} ; \quad a_{j} \in \mathbb{R}\right\}
$$

se tiene:

$$
A G(2) \equiv A G_{1}(2) \oplus A G^{+}(2)
$$

y podemos concluir:

- Identificando $\quad u_{1}$ y $u_{2}$ con la base canónica de $\mathbb{R}^{2}$, explicitamos que AG(2) contiene una copia isomorfa de $\mathbb{R}^{2}$, como $\mathbb{R}$-subespacio vectorial.

- Como $\left(u_{1} u_{2}\right)\left(u_{1} u_{2}\right)=-1$, usando la identificación $u_{1} u_{2} \equiv \imath \in \mathbb{C}$, explicitamos que $\mathrm{AG}(2)$ contiene una copia isomorfa de los números complejos $\mathbb{C}$, como $\mathbb{R}$-subálgebra conmutativa.

- Los resultados anteriores determinan un isomorfismo, como $\mathbb{R}$-espacios vectoriales:

$$
A G(2) \equiv \mathbb{C} \oplus \mathbb{R}^{2} \text {. }
$$

Con este isomorfismo la tabla del producto euclideano (3.1) permite verificar los tres resultados algebraicogeométricos que enunciamos a seguir:

(para verificar se puede usar $v=a u_{1}+b u_{2}, \quad w=c u_{1}+d u_{2} \quad$ y $\quad z=r+s u_{1} u_{2} \equiv r+s i$ ).

1. En el Álgebra Geométrica AG(2) se cumple la Identidad Geométrica de Euler:

El producto euclideano de los vectores no nulos $v, w \in \mathbb{R}^{2}$, es el número complejo

$$
v w=\|v\|\left\|\left|w\left\|\mathfrak{e}^{\theta \imath}=\right\| v\|\|\right| w\right\| \cos \theta+\||| v|\||| w\|(\sin \theta) \imath
$$

cuyo módulo es $|v w|=\|v\|\|\mid w\|$ y cuyo argumento $\theta=\mu \varangle(v, w) \in]-\pi, \pi]$ es la medida del ángulo orientado de $v$ a $w$ (consideraremos $\theta=\pi$ si y solo si $v$ y $w$ tienen sentidos opuestos; es decir, $v\|w\|+$ $w\|v\|=0)$.

Reciprocamente, todo número complejo es el producto euclideano de dos vectores, pero sin unicidad de los mismos.

2. Como $-\theta \in]-\pi, \pi]$ es la medida del ángulo orientado de $w$ a $v$, resulta que $w v$ es el complejo conjugado de $v w$ dado en (3.5), precisamente:

$$
w v=\|w\|\|v\| \mathfrak{e}^{-\theta \imath}=\|w\|\|v\| \cos \theta-\|w\|\|v\|(\sin \theta) \imath
$$

3. Los productos euclideanos, $w z$ y $z w$, de un vector $w$ con un complejo $z$, son vectores simétricos respecto de $w$ que resultan de rotar el vector $|z| w$ (una homotecia de $w$ ) el ángulo orientado $\pm \arg (\mathrm{z})$ respectivamente, como sugiere el siguiente diagrama (con $|z|>1$ y $0<\arg (z)<\frac{\pi}{2}$ ):

La Identidad Geométrica de Euler (3.5) es llamada así porque, considerando $v$ y $w$ unitarios la segunda igualdad de (3.5) se reduce a la identidad de Euler

$$
e^{\theta \imath}=\cos \theta+(\sin \theta) \imath ; \theta \in \mathbb{R},
$$

que tiene, como caso particular, a la famosa Ecuación de Euler

$$
e^{\pi \imath}+1=0
$$

conocida desde antes de 1748 y sobre la cual, en 1988, la revista Mathematical Intelligencer dio a conocer que un selecto grupo de matemáticos había opinado que era el resultado más hermoso de toda la matemática (ver [6]). 


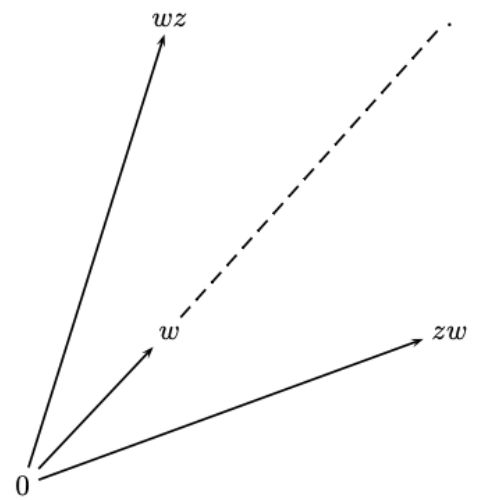

FIGURA 3.1. Productos euclideanos de vector con complejo

4. Algebrizando la Geometría. Veamos algunos ejemplos de como la estructura del álgebra geométrica AG(2) permite algebrizar algunos conceptos matemáticos:

4.1. La métrica euclideana del plano. $\mathbb{R}^{2}=\left\{a_{1} u_{1}+a_{2} u_{2} ; \quad a_{j} \in \mathbb{R}\right\}$ es considerado, de inicio, tan solo como un espacio vectorial. Utilizando el producto euclideano de $\mathrm{AG}(2)$ se tiene:

$$
v=v_{1} u_{1}+v_{2} u_{2} \in \mathbb{R}^{2} \Longrightarrow v v=v_{1}^{2}+v_{2}^{2}
$$

es decir, definiendo

$$
\|v\|:=\sqrt{v v} .
$$

tenemos que el producto euclideano determina la métrica euclideana en $\mathbb{R}^{2}$.

4.2. Ortogonalidad y colinealidad de vectores. Sumando y restando las identidades geométricas de Euler (3.5) y (3.6) algebrizamos de modo invariante (sin usar coordenadas) los conceptos geométricos de ortogonalidad y colinealidad de vectores; en efecto, dados los vectores no nulos $u, v \in \mathbb{R}^{2}$ :

- $u v+v u=2\|u|\|||v| \cos \theta=2 u . v, \quad$ permite redefinir el producto interior en el contexto de $\mathrm{AG}(2)$

$$
u . v:=\frac{u v+v u}{2}
$$

y con esto algebrizar la ortogonalidad de vectores en $\mathrm{AG}(2)$, del siguiente modo:

$$
u \perp v \Longleftrightarrow u v+v u=0 \text { (su producto euclideano anticonmuta). }
$$

- $u v-v u=2\|u\|\|\mid v\|(\sin \theta) \imath \in A G(2)$, permite definir el producto exterior de dichos vectores

$$
u \uparrow v:=\frac{u v-v u}{2}=\|u\|\|v\|(\sin \theta) \imath
$$

que representa algebraicamente, en $\mathrm{AG}(2)$, el área del paralelogramo que determinan los vectores $u \mathrm{y} v$, porque su módulo $|u \uparrow v|=\| u|||v||| \sin \theta \mid$ es igual al área del paralelogramo que determinan dichos vectores. Esto permite algebrizar la colinealidad de vectores en $\mathrm{AG}(2)$, del siguiente modo:

$$
u \| v \Longleftrightarrow u v-v u=0 \text { (su producto euclideano conmuta). }
$$

4.3. El concepto de base ortonormal. De lo anterior resulta que la versión algebraica del concepto de base ortonormal está dada por las condiciones de Grassmann-Clifford:

$$
u_{1} u_{1}=u_{2} u_{2}=1 \quad \text { y } \quad u_{2} u_{1}+u_{1} u_{2}=0 .
$$

Esto se sustenta en las identidades (4.1) y (4.2) porque $\left\|u_{1}\right\|=u_{1} u_{1} \quad$ y $\quad\left\|u_{2}\right\|=u_{2} u_{2}$.

4.4. Construcción de bases ortonormales orientadas. Como $\arg (\imath)=\frac{\pi}{2}$, dado un vector unitario $u \in$ $\mathbb{R}^{2} \backslash\{0\}$ podemos construir bases ortonormales orientadas: $(u, u \imath)$ positivamente orientada y $(u, \imath u)$ negativa-

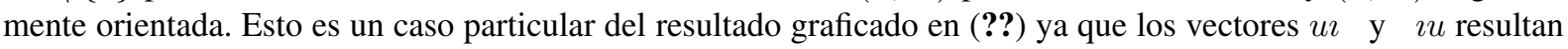
ortogonales a $u$, de la misma longitud de $u$ y uno inverso del otro, precisamente: 


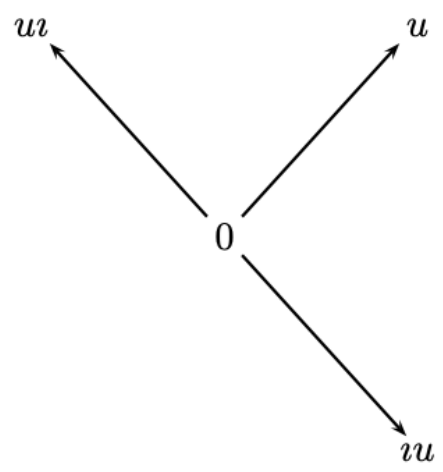

FIGURA 4.1. Bases ortonormales orientadas

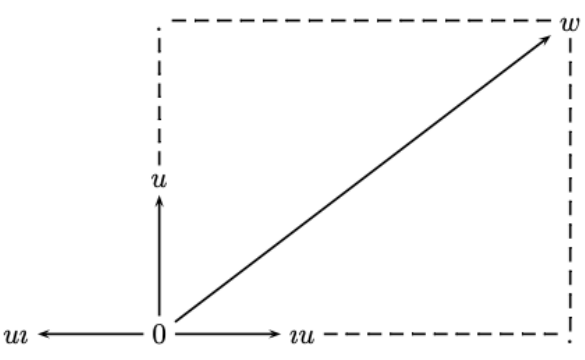

4.5. Descomposición ortogonal. Dados $u, w \in \mathbb{R}^{2} \backslash\{0\}$, con $u$ unitario, obtenemos algebraicamente, en el contexto de $\operatorname{AG}(2)$, las descomposiciones ortogonales del vector $w$ respecto de las bases ortonormales dadas en el diagrama (??), precisamente:

$$
w=(w \cdot u) u+(w \cdot(u \imath)) u \imath=[(w \cdot u) u+(w \cdot(-u \imath))(-u \imath)]=(w \cdot u) u+(w \cdot(\imath u)) \imath u
$$

4.6. El concepto de reflexión. Consiste en calcular algebraicamente el vector $r_{u}(w)$, reflexion del vector $w \in \mathbb{R}^{2} \backslash 0$ respecto de una recta $l$ que pasa por el origen y es ortogonal al vector unitario $u \in S^{1}$,

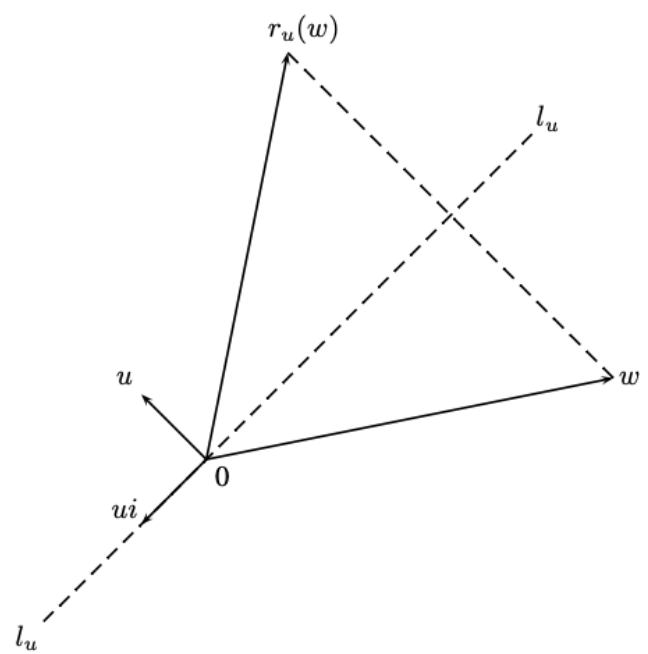

Recordemos que geométricamente se utiliza el siguiente argumento:

$$
w=(w \cdot u) u+(w \cdot(u \imath)) u \imath \Longrightarrow r_{u}(w)=-(w \cdot u) u+(w \cdot(u \imath)) u \imath
$$

Algebraicamente, en el contexto de $\mathrm{AG}(2)$, se utilizan los siguientes ingredientes:

- $-\imath u=u \imath \quad$ у $\quad u u=1$.

- w.u y $w \cdot(u \imath)$, como escalares, conmutan con todo número geométrico. 
- De lo anterior, $u w u=u[(w \cdot u) u+(w \cdot(u \imath)) u \imath] u=(w \cdot u) u-(w \cdot(u \imath)) u \imath \in \mathbb{R}^{2}$.

- La aplicación de reflexión, respecto de la recta $l_{u}$ que pasa por el origen y es ortogonal a $u \in S^{1}$,

$$
\rho_{u}: w \in \mathbb{R}^{2} \longmapsto-u w u \in \mathbb{R}^{2} \text {, está bien definida. }
$$

Usando estos ingredientes y la primera descomposición de $w$ dada en (4.4) se concluye:

$$
\rho_{u}(w)=-u w u=-u[(w \cdot u) u+(w \cdot(u \imath)) u \imath] u=-(w \cdot u) u+(w \cdot(u \imath)) u \imath=r_{u}(w) .
$$

4.7. El concepto de rotación. Este importante resultado es consecuencia inmediata de las identidades geométricas de Euler (3.5) y (3.6) y el siguiente teorema clásico de la geometría euclideana que caracteriza las rotaciones en términos de reflexiones (ver, por ejemplo, la referencia [1])

La composición de dos reflexiones determinan una rotación

$$
\text { y recíprocamente, }
$$

toda rotación se descompone de ese modo, sin unicidad de las reflexiones.

En concordancia con el enfoque dado a las reflexiones, algebrizaremos rotaciones centradas en $0 \in \mathbb{R}^{2}$.

Proposition 4.1. Dadas las reflexiones $\rho_{u} y \rho_{v}$, del tipo dado en (4.6), donde $u, v \in S^{1}$ son linealmente independientes y $\theta=\mu(\varangle(u, v)) \in]-\pi, \pi[\backslash\{0\}$ es la medida del ángulo orientado de u a $v$, entonces

$$
\varrho_{2 \theta}=\rho_{v} \rho_{u}: w \in \mathbb{R}^{2} \longmapsto v u w u v=\mathfrak{e}^{-\theta \imath} w \mathfrak{e}^{\theta \imath} \in \mathbb{R}^{2}
$$

es la aplicación que algebriza la rotación orientada en el ángulo orientado $2 \theta$.

Recíprocamente, toda rotación orientada en el ángulo orientado $2 \theta$ se descompone del modo indicado en la identidad (4.7), sin unicidad de los vectores unitarios considerados.

La rotación orientada en el ángulo orientado $-2 \theta$, es algebrizada por la aplicación

$$
\varrho_{-2 \theta}(w)=\rho_{u} \rho_{v}(w)=u v w v u=\mathfrak{e}^{\theta \imath} w \mathfrak{e}^{-\theta \imath} .
$$

Las expresiones (4.7) y (4.8) son una inversa de la otra y resultan familiares en el ambiente físico por su relación con los espinores bidimensionales.

Después de la primera reflexión, $\rho_{u}$, se tiene el gráfico (??).

Luego de la segunda reflexión $\rho_{v}$, se obtiene la rotación (4.7) $\varrho_{2 \theta}=\rho_{v} \rho_{u}$ cuyo gráfico es:

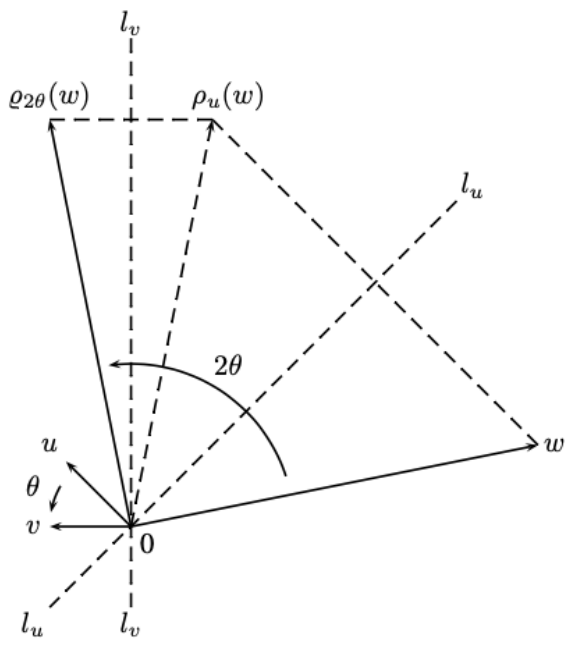

Tomar nota que $2 \theta=\mu \varangle\left(w, \varrho_{2 \theta}(w)\right)$ y que en la figura estamos considerando $0<\theta<\frac{\pi}{2}$.

\section{Conclusiones.}

- AG(2) optimiza el álgebra de matrices $\mathbb{R}^{2 \times 2}$, una subálgebra de aquella que Pauli utilizó en los inicios de la mecánica cuántica para su teoría del electrón, siguiendo lo recomendado por Heisenberg en 1920:

La Física requiere una matemática completamente nueva que incluya álgebras no conmutativas.

- Ofrecemos material amigable para adoptar lo recomendado por Félix Klein en su Erlanger Programm de 1908: 
Un docente puede abordar con mayor solvencia y convicción el tema que le corresponda transmitir a sus alumnos, estudiándolo previamente desde un punto de vista avanzado.

Klein plasmó sus ideas en el libro para docentes preuniversitarios de matemática

Matemática básica desde un punto de vista superior ([4] y [5]),

una visión unificada utilizando la matemática desarrollada hasta el siglo XIX.

- Se amplía el conocido encaje de álgebras reales $\mathbb{R}<\mathbb{C}$ al encaje

$$
\mathbb{R}<\mathbb{C}<A G(2) .
$$

- Existe una relación biyectiva entre Espinores bidimensionales y complejos:

$$
\mathbb{R}^{2} \ni\left(r_{1}, r_{2}\right) \leftrightarrow r_{1}+r_{2} i \in \mathbb{C} .
$$

Es justamente lo que hizo Hamilton al formalizar los complejos.

- Existe un isomorfismo de grupos entre U(1) y los complejos unimodulares.

$$
U(1) \ni\left(\begin{array}{cc}
\cos \theta & -\sin \theta \\
\sin \theta & \cos \theta
\end{array}\right) \leftrightarrow \mathfrak{e}^{\theta \imath} \in \mathbb{C} .
$$

- Las bondades anteriores y sus aplicaciones en diversas áreas, (ver [7]), refuerzan la propuesta de incluir el Algebra Geométrica como parte de la Matemática Básica para docentes y alumnos universitários de Física y Matemática del siglo XXI.

Agradecimientos. Reiteramos nuestro agradecimineto al Comité Editor de SELECCIONES MATEMÁTICAS por su amable acogida, en particular, al Dr. Obidio Rubio por su gentil apoyo.

ORCID and License

Edgar Vera Saravia https://orcid.org/0000-0002-3634-8549.

This work is licensed under the Creative Commons Attribution-NoComercial-ShareAlike 4.0.

Referencias

[1] Barker - Howe Continuous Symmetry (From Euclid to Klein); American Mathematical Society, 2007.

[2] Hestenes, D. Space-Time Algebra; Gordon and Breach, 1966.

[3] Hestenes, D. New Foundations for Classical Mechanics; Kluwer Academic Publishers, 1993.

[4] Klein, F. Elementary Mathematics from an advanced standpoint, Arithmetic-Algebra-Analysis; The Macmillan Company, 1932.

[5] Klein, F. Elementary Mathematics from an advanced standpoint Geometry; The Macmillan Company, 1939.

[6] Nguyen L. http://www.science4all.org/le-nguyen-hoang/eulers-identity/; Science4all, 2016.

[7] Prewass, C. Geometric Algebra with Applications in Engineering; Springer, 2009.

[8] Vera - Zubieta Sobre el espacio euclideano bidimensional; Selecciones Matemáticas, Vol. 05(02), 2018. 